\title{
Alquiler vacacional en destinos turísticos consolidados: la venganza de los actores que ya no importan

\author{
Yurena Rodríguez Rodríguez***
} \\ Josefa Rosa Marrero-Rodríguez* Moisés Simancas Cruz** \\ Universidad de La Laguna (España)
}

\begin{abstract}
Resumen: En este trabajo se atiende al crecimiento del alquiler vacacional en los destinos turísticos consolidados, tomando la isla de Tenerife como caso de estudio. En concreto, se analiza la influencia de la estructura alojativa actual en el proceso de expansión de este modelo de negocio; y, por otro lado, el impacto que el alquiler vacacional pueda tener sobre las reglas del juego en el campo turístico. Se parte de una perspectiva teórica que analiza los espacios económicos como realidades productivas complejas, conformadas por actores y capitales, que interactúan en bases a pautas comportamentales fundamentadas en normas y (des)equilibrios de poder. Metodológicamente se procede mediante una aproximación coherente, sistemática y triangular de distintas fuentes de datos. En cuanto a los resultados, se observa un incremento del alquiler vacacional, condicionado parcialmente al modelo turístico ya existente, y también un debate abierto y conflictivo relativo a las normas de funcionamiento en el sector.
\end{abstract}

Palabras Clave: Alquiler vacacional; Campos de acción estratégico; Turismo; Tenerife; Recursos; Poder.

\section{The vacation rental in the consolidated tourist destinations: revenge of unimportant actors}

Abstract: The objective of this research is the vacation rental growth in tourist destinations; Tenerife Island is taking like a case study. Specifically, we analyzed the influence of the accommodation structure on the expansion process of this business model; and, on the other hand, the impact that the vacation rental may have on the rules of the game in the tourist field. It starts from a theoretical perspective that analyzes economic spaces as complex productive realities, made up of actors and capitals, which interact on the basis of behavioral guidelines based on norms and (un) equilibria of power. Methodologically, we proceed by means of a coherent, systematic and triangular approach from different data sources. As for the results, there is an increase in vacation rental, partially conditioned by the existing tourism model, and also an open and conflicting debate regarding the operating rules in the sector.

Keywords: Vacation rental; Strategic action fields; Tourism; Tenerife; Resources; Power.

\section{Introducción}

El análisis de los espacios económicos como realidades productivas complejas, conformadas por actores, capitales y reglas de juego es fructífero desde el punto de vista teórico y empírico. En este trabajo se atiende al crecimiento del alquiler vacacional en los destinos turísticos consolidados, tomando como estudio de caso la isla de Tenerife. En concreto se abordan la influencia del modelo turístico insular en la expansión del alquiler vacacional y los cambios discursivos sobre qué entender por espacio turístico ${ }^{1}$.

La orientación teórica adoptada analiza los destinos turísticos como realidades productivas o entornos ecológicos conformados por empresas, administraciones y stakeholders. En cuanto al abordaje metodológico, se procede mediante una aproximación coherente, sistemática y triangular de distintas fuentes de datos (Bericat, 1998). En los campos económicos, los actores implicados se valen de estra-

\footnotetext{
* Universidad de La Laguna (España); E-mail: jrmarrod@ull.edu.es; https://orcid.org/0000-0002-9542-4003

** Universidad de La Laguna (España); https://orcid.org/0000-0002-0337-0424

*** Universidad de La Laguna (España); https://orcid.org/0000-0002-3258-9898
} 
tegias para alcanzar la innovación, el reposicionamiento frente a otros competidores, y la mejora de la rentabilidad. En el caso de los destinos turísticos, estas acciones pueden ser la renovación de los alojamientos turísticos, la búsqueda de nuevos mercados y consumidores, la acción sobre los medios de transporte y comercialización, los cambios organizativos y tecnológicos, entre otros. Las acciones se implementan con diferentes herramientas e intensidad. Así, asumimos que el entramado productivo turístico es complejo y heterogéneo, a la vez que resultado de su historia reciente.

En la última década, el alquiler vacacional se ha desarrollado rápidamente en España, también en espacios donde el alojamiento que denominaremos convencional (hoteles y apartamentos) estaba consolidado. En tanto el alquiler vacacional se está extendiendo a gran velocidad por ciudades y países, cabe pensar que dicha expansión encuentra condicionantes y estímulos específicos en cada uno de los espacios.

\section{Planteamiento teórico, objetivos, hipótesis y metodología}

Es fructífero estudiar el sector turístico como un campo compuesto por actores diversos en cuanto a recursos y actitudes, y donde la historia es central para comprender las relaciones más o menos equilibradas que mantienen en cada momento. Partimos principalmente de la teoría de los campos de acción estratégica, que combina el análisis bourdiano con las ideas de Giddens y la nueva teoría institucional en el estudio de las organizaciones (Fligstein y McAdam, 2011; 2012). Los campos son espacios de interacción individual y colectiva, que funcionan en base a reglas y valores. Están jerarquizados, en permanente cambio e influidos por dinámicas internas y externas, procedentes de otros campos (organizaciones, instituciones, Estado).

En el campo de acción estratégico, los actores compiten por obtener ventaja, derivada del uso de los diferentes recursos disponibles (Fligstein, 2013), convirtiendo a las relaciones de poder en una variable importante. Es frecuente que existan dos colectivos, los miembros titulares y los que desafían a aquellos en sus posiciones. Los límites del campo de acción estratégico no son fijos, cambian con el tiempo. El campo obtiene estabilidad a partir de posiciones estables, y de la existencia de unidades de gobierno, encargadas de supervisar el cumplimiento de las normas y el buen funcionamiento del sistema. En dichos campos es importante la existencia de definiciones colectivas, mínimamente consensuadas, relativas a lo que es importante y qué no, a los actores legítimos para ocupar posiciones de poder y prestigio, o con respecto a los objetivos de la organización. No siempre hay consenso, por lo que son frecuentes las luchas por la definición de las normas reguladoras del campo. Esta propuesta teórica ha sido adoptada teniendo en cuenta las matizaciones de mejora expuestas por Goldstone y Useem (2012).

La teoría de los campos de acción estratégica presenta algunas afinidades con otras aproximaciones analíticas relativas a los destinos turísticos. Así, para el caso de los modelos evolutivos descriptivos-predictivos de los destinos, Butler (2006; 2012) y Butler y Russell (2010) han reconocido que en éstos puede haber subespacios con momentos de desarrollo diferenciados; esto no estaba presente en la famosa propuesta originaria de Butler (1980). También hay afinidad con la teoría de la regulación o la geografía económica evolutiva, salvo que la propuesta de Fligstein y McAdam permite operacionalizar mejor los recursos y actores implicados, las diferencias entre ellos y, por tanto, las oportunidades o dificultades que encuentran (Agarwal, 1997; 2002; 2006; Agarwal y Shaw, 2007; Cooper y Jackson, 1989; Garay y Cànoves, 2011; Ma y Hassink, 2013; Oppermann, 1998; Oreja et al., 2008; Ravenscroft y Hadjihambi, 2006). El concepto de campo económico visibiliza el sistema productivo tal que los distintos actores presentan cantidades diferenciales de recursos, y esto desemboca en un mapa de racionalidades no siempre coincidentes, y en alianzas más o menos factibles dentro del campo.

A partir de esta orientación teórica, el objetivo general es analizar la influencia del campo económico turístico actual en el mapa expansivo del alquiler vacacional y en qué medida los nuevos actores implicados en este modelo de negocio reajustan las normas de funcionamiento de dicho campo. De forma más concreta, los subobjetivos en este artículo son la influencia de la oferta turística alojativa convencional en el mapa resultante de alquiler vacacional, y en qué medida éste puede cambiar las reglas del juego en el campo, al reconceptualizar algunas de sus reglas de funcionamiento.

Y las hipótesis son dos. En primer lugar, el alquiler vacacional aprovecha en Tenerife los espacios alojativos turísticos consolidados, especialmente los alojamientos extrahoteleros. Y, en segundo lugar, los actores promotores del alquiler vacacional buscan cambiar las reglas del juego, en tanto se acepten las definiciones de espacio turístico que proponen.

La aplicación de esta propuesta teórica al espacio turístico de Tenerife se hace con las siguientes precisiones conceptuales. En primer lugar, las estrategias empresariales. Nos centraremos en una de ellas, la apuesta por el alquiler vacacional, pero que necesariamente debe tener en cuenta el alojamiento 
convencional (expresión que se utilizará de aquí en adelante, y que se refiere a hoteles, complejos extrahoteleros, hoteles rurales, camping y cruceros), con el que compite. Por tanto, la atención se centra en el conjunto de empresas alojativas. La llegada del alquiler vacacional introduce en el campo a un cuarto actor, los emprendedores y promotores del alquiler vacacional.

No obstante, en segundo lugar, este campo de acción estratégica está influido e interseccionado por dos campos más. Por un lado, el Estado, cuyo poder normativo condiciona la acción empresarial; en este caso por la acción reguladora de la administración autonómica (Fligstein y McAdam, 2011). Por otro lado, el sistema turístico europeo. La mayor parte de los turistas que visitan el Archipiélago Canario proviene de algún país de la Unión Europea; por ello, las compañías aéreas y los Tour Operadores (TO) son centrales para comprender la dinámica en el Archipiélago. El destino Canarias constituiría un subespacio dentro del sistema turístico europeo. De esta forma, Estado y TO son dos stakeholders destacados, y esto es fundamental para comprender que los cambios adoptados por las empresas alojativas responden al menos parcialmente a su acción reguladora y/o capacidad de influencia (Marrero-Rodríguez, 2019).

En tercer lugar, los recursos disponibles. Al tratarse de un campo económico, los dos recursos centrales son los financieros y profesionales. No obstante, los mecanismos de comercialización y la acción normativa también son importantes. Y estos cuatro tipos de recursos están desigualmente distribuidos. La importancia de los mecanismos de comercialización favorece tradicionalmente a los TO debido a su cercanía al cliente (también a las compañías aéreas), la regulación del destino a las administraciones, y los capitales a las empresas. Pero al interior de cada actor, existen diferencias jerarquizantes. Así, las grandes empresas alojativas hoteleras cuentan con más capitales financieros, profesionales y de plazas alojativas, lo que favorece su influencia en las negociaciones con el resto de stakeholders.

El alquiler vacacional activa un mecanismo de comercialización que no pasa por el TO sino por las plataformas digitales de reserva de alojamiento (Airbnb, HomeAway, Niumba, Wimdu, Housetrip, Rentalia, BeMate, Only-Apartments). Dichas plataformas son un modelo de negocio que genera valor a través de una relación directa entre oferentes y consumidores, en un proceso caracterizado por algunos autores como innovación disruptiva (Bashir y Verma, 2016; Guttentag, 2015; Mikhalkina y Cabantous, 2015; Onete et al., 2018). Dado que la gestión de la comercialización sigue estando en Canarias condicionada en gran medida por los TO, y al aportar el alquiler vacacional una fórmula de comercialización de sencilla implementación, pasa a competir con el TO y canales de los alojamientos convencionales, y finalmente obliga a reordenar las relaciones entre actores en el campo. Todo esto ha dado lugar a una discusión académica sobre el impacto del alquiler vacacional a la que este texto no es ajeno (Garau-Vadell et al., 2019; Groizard y Nilsson, 2017; Hall y Müller, 2018; Perles Ribes et al., 2018; Yrigoy, 2017).

A partir de este marco teórico, precisiones conceptuales, objetivos e hipótesis, se describe la metodología empleada. Para el estudio de la oferta turística alojativa tinerfeña se utilizan los datos publicados por el departamento de Desarrollo Económico de Turismo de Tenerife (Cabildo Insular de Tenerife, www. webtenerife.com/investigacion/) y la Encuesta de Alojamiento Turístico (Instituto de Estadística de Canarias, ISTAC). En cuanto a la oferta de alquiler vacacional, se ha tomado como referencia los datos publicados por el Observatorio del Turismo de Canarias, perteneciente a la Viceconsejería de Turismo del Gobierno de Canarias; en este caso, la información sobre la procedencia de los datos relativos al alquiler vacacional es la siguiente: "generar un universo propio de datos a través de la consulta de información accesible públicamente en las plataformas digitales"; por tanto, los datos proceden de una búsqueda en 2018 de la oferta publicada en los portales de Airbnb, Homeaway, Niumba, Atraveo, Rentalia, Booking, 9flats y Onlyapartments. También, de forma complementaria se han utilizado los datos procedentes de DataHippo. Éste es un proyecto colaborativo que ofrece datos de diferentes plataformas de alquiler turístico; la consulta se realizó en noviembre de 2019. En el caso de DataHippo y del Observatorio del Turismo de Canarias, la técnica empleada para cuantificar el alquiler vacacional es la denominada web-scrapping (Wegmann y Jiao, 2017; Yrigoy, 2017). Siempre que sea posible, los datos harán referencia a 2019; sin embargo, a veces la referencia será 2018, con el objeto de conservar la equivalencia con el informe sobre alquiler vacacional publicado por el Observatorio del Turismo de Canarias.

En cuanto al contexto normativo y social, se ha estudiado la legislación turística autonómica más relevante de los últimos 30 años. Para analizar los discursos de los actores se ha realizado una doble aproximación con once entrevistas en profundidad a empresarios y directivos de empresas alojativas, responsables de estrategias públicas de modernización turística y a directivos de la patronal turística de la provincia de Santa Cruz de Tenerife (Ashotel) -once entrevistas-, declaraciones de representantes de la Asociación Canaria de Alquiler Vacacional (ASCAV) y también con un análisis complementario de la prensa local. Con respecto a esto último, se ha procedido mediante una búsqueda de noticias relacionadas con la expresión 'Tenerife' y 'alquiler vacacional' desde el 1 de julio de 2018 hasta el febrero de 2020, atendiendo a actores y temas. 


\section{La oferta turística alojativa de Tenerife}

Sea en el ámbito europeo o en el español, el sector turístico canario es muy importante. En líneas generales, se caracteriza por estar fundamentado en el volumen, una posición subordinada en la cadena de valor turística europea de sol y playa, y por explotar sobre todo recursos físicos y naturales (Hernández Martín, 2016). Todo ello, en un contexto de debilidad de los impactos económicos del turismo, dada la dificultad para convertirse en elemento motriz a nivel insular (Padrón-Marrero y Rodríguez-Martín, 2015).

Según el Instituto Canario de Estadística, la isla de Tenerife recibió 5.729.162 turistas en 2019. La mayor parte del alojamiento se concentra en tres municipios (77,8\% de plazas alojativas convencionales): Puerto de la Cruz es el núcleo más antiguo, situado en el norte insular; Adeje y Arona, a partir de los 70-80, en el sur. En las últimas décadas, beneficiéndose de la expansión turística, destacan otros municipios alrededor de Arona y Adeje (Guía de Isora, Santiago del Teide, San Miguel de Abona y Granadilla de Abona). Todos los municipios mencionados suman el 89,7\% de las plazas alojativas convencionales. En la isla tinerfeña se contabilizaron en 2018, 149.744 plazas alojativas convencionales y 54.168 de alquiler vacacional -tabla I- (Observatorio del Turismo de Canarias).

Tabla I: Relación de plazas de vivienda vacacional, plazas de oferta reglada, precio medio y ADR según municipios, 2018

\begin{tabular}{|c|c|c|c|c|c|}
\hline & Plazas VV & $\begin{array}{l}\text { Plazas oferta } \\
\text { reglada }\end{array}$ & $\begin{array}{c}\text { Precio medio } \\
\text { habitaciones VV }\end{array}$ & $\begin{array}{c}\mathrm{ADR} \\
\text { apartamentos }\end{array}$ & $\begin{array}{c}\text { ADR } \\
\text { hoteles }\end{array}$ \\
\hline Isla de Tenerife & 54.168 & 149.744 & 38,4 & 65,9 & 95,1 \\
\hline Adeje & 10.455 & 50.412 & 40,6 & 67,0 & 116,1 \\
\hline Arona & 9.859 & 43.369 & 52,0 & 69,8 & 95,2 \\
\hline Granadilla de Abona & 3.993 & 2.626 & 25,4 & 69,6 & \\
\hline Santiago del Teide & 2.198 & 7.402 & 55,8 & 71,3 & 109,0 \\
\hline Puerto de la Cruz & 2.277 & 23.148 & 38,4 & & 53,2 \\
\hline Santa Cruz de Tenerife & 3.265 & 4.165 & 28,2 & 43,2 & 64,1 \\
\hline San Miguel de Abona & 2.323 & 4.800 & 70,1 & & \\
\hline San Cristóbal de La Laguna & 2.415 & 1.690 & 26,2 & & \\
\hline Arico & 1.859 & 562 & 32,8 & & \\
\hline Guía de Isora & 1.937 & 3.204 & 31,5 & & \\
\hline La Orotava & 1.444 & 422 & 60,5 & & \\
\hline Los Realejos & 957 & 2.062 & 69,8 & & \\
\hline Tacoronte & 901 & 567 & 34,3 & & \\
\hline Candelaria & 1.313 & 1.428 & 31,7 & & \\
\hline Santa Úrsula & 830 & 1.090 & 34,0 & & \\
\hline El Rosario & 977 & 407 & 34,2 & & \\
\hline Güimar & 1.087 & 472 & 58,8 & & \\
\hline La Matanza de Acentejo & 609 & 220 & 47,5 & & \\
\hline Garachico & 398 & 368 & 28,0 & & \\
\hline Buenavista del Norte & 360 & 386 & 28,3 & & \\
\hline El Sauzal & 452 & 201 & 20,8 & & \\
\hline Fasnia & 271 & 69 & 250,0 & & \\
\hline San Juan de la Rambla & 282 & 102 & 48,6 & & \\
\hline Arafo & 210 & 56 & 19,4 & & \\
\hline El Tanque & 303 & 108 & 24,3 & & \\
\hline Tegueste & 347 & 121 & 28,6 & & \\
\hline Los Silos & 233 & 276 & 59,8 & & \\
\hline La Guancha & 216 & 87 & 80,0 & & \\
\hline Vilaflor & 156 & 212 & 49,8 & & \\
\hline La Victoria de Acentejo & 132 & 27 & 23,3 & & \\
\hline
\end{tabular}

Fuente: Observatorio de Turismo de Canarias. 
En primer lugar, destaca el importante crecimiento de la oferta alojativa (gráfico 1). Éste es un destino turístico consolidado. La opción de creación de nuevos establecimientos se encuentra limitada desde hace años por la normativa autonómica; sin embargo, se observa un repunte a partir de 2018, que tiene que ver con la llegada del alquiler vacacional. En segundo lugar, a lo largo de las décadas se observa una tendencia sostenida de reconfiguración de la oferta alojativa. Desde un equilibrio entre plazas extrahoteleras y hoteleras, a un predominio de las segundas. A ello han contribuido la normativa y la mayor rentabilidad de los establecimientos hoteleros (véase tabla II, gráficos 2 y 3). Cada vez más destacan los alojamientos de calidad media-alta: los hoteles de 4 y 5 estrellas representan el $29,1 \%$ del total de establecimientos y el 50\% de las plazas de la isla (Encuesta Alojamiento Turístico, ISTAC, año 2019). No obstante, el $34 \%$ del total de plazas son extrahoteleras.

\section{Gráfico 1: Evolución del número de plazas turísticas en Tenerife, 1980-2018}

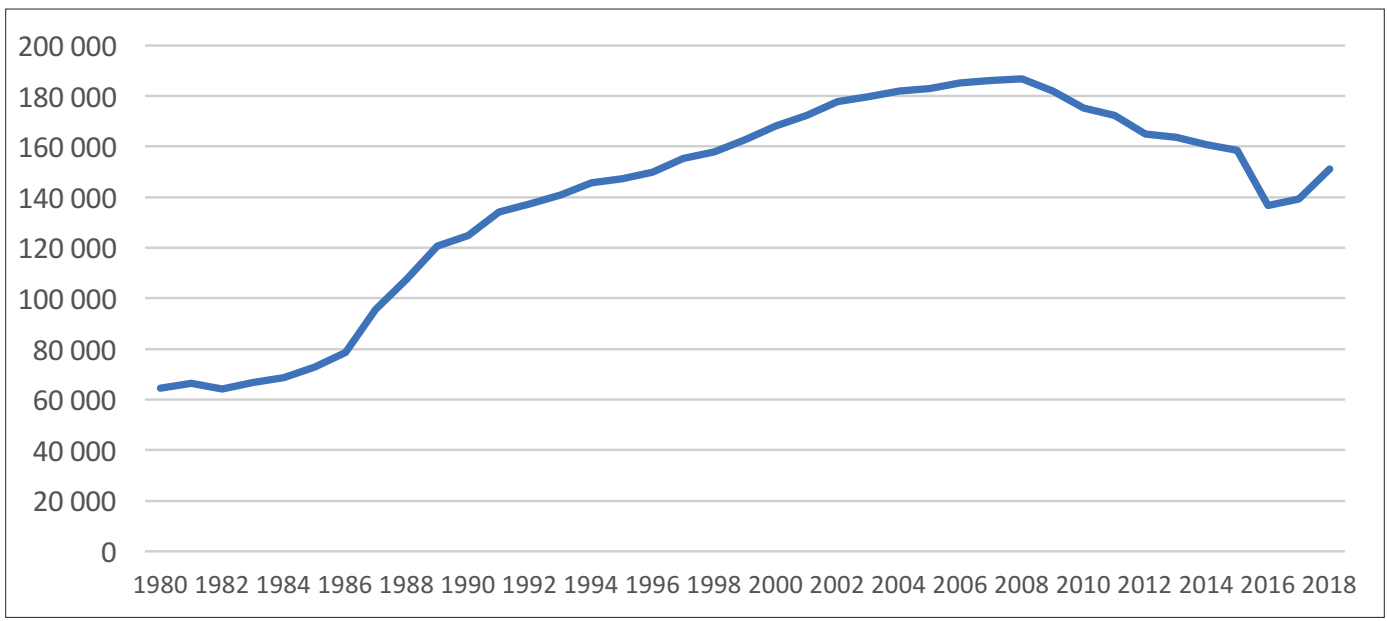

Fuente: Desarrollo Económico, Cabildo Insular de Tenerife, Turismo de Tenerife.

Tabla II: Resumen de indicadores de oferta y demanda en alojamientos hoteleros y extrahoteleros de Tenerife 2019

\begin{tabular}{|l|c|c|}
\hline \multicolumn{1}{|c|}{ Indicador } & Hotelero & Extrahotelero \\
\hline Tasa de ocupación (por plazas) & $75,09 \%$ & $59 \%$ \\
\hline Ingreso por habitación disponible (REVPAR) & $76,4 €$ & $50,7 €$ \\
\hline Porcentaje de pernoctaciones (\% horizontal) & $69,6 \%$ & $30,3 \%$ \\
\hline Viajeros alojados (\% horizontal) & $72,5 \%$ & $27,4 \%$ \\
\hline Plazas (\% horizontal) & $65,6 \%$ & $34,3 \%$ \\
\hline
\end{tabular}

Fuente: Encuesta de Alojamiento Turístico, ISTAC. 
Gráfico 2: Evolución de las plazas hoteleras y extrahoteleras en Tenerife, 1980-2018

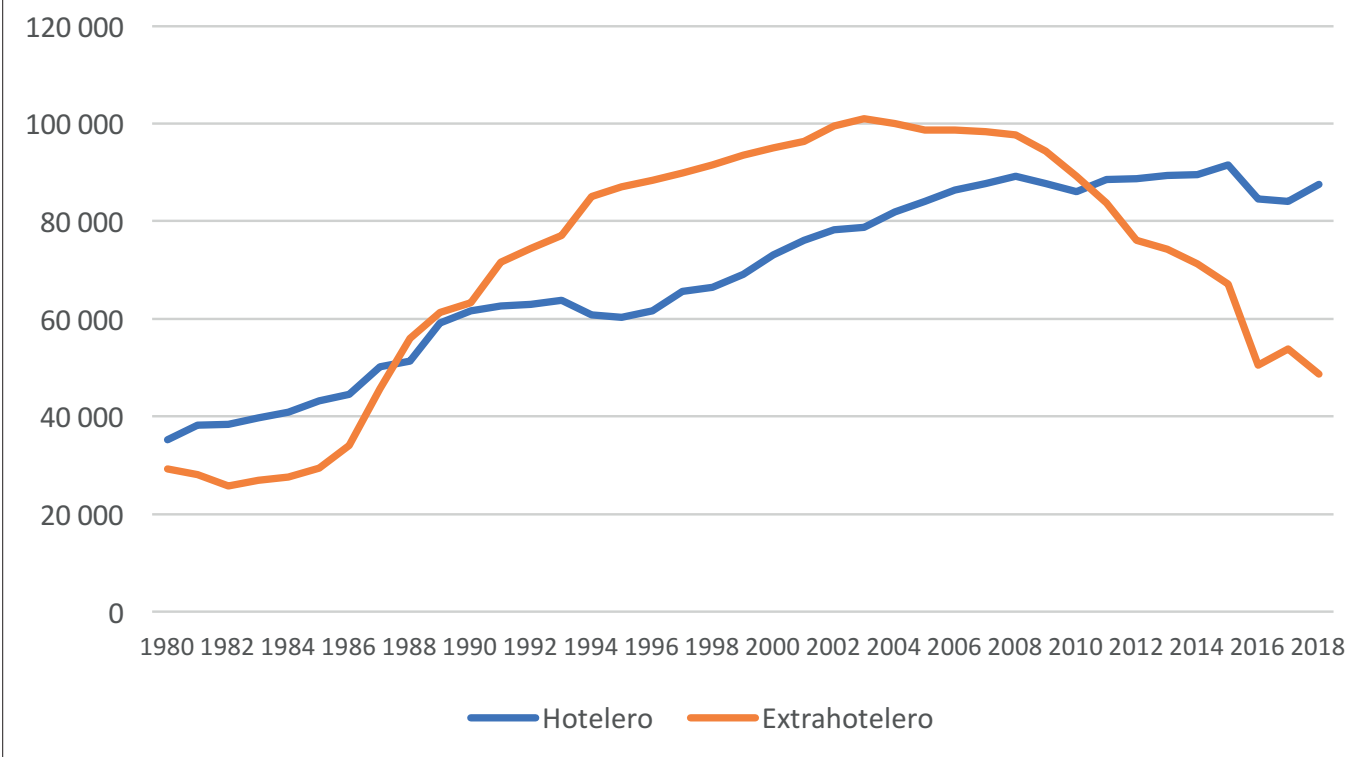

Fuente: Desarrollo Económico, Cabildo Insular de Tenerife, Turismo de Tenerife.

Gráfico 3. Plazas turísticas ofertadas según tipología del establecimiento, 2019



Fuente: Encuesta de Alojamiento Turístico, ISTAC.

En tercer lugar, las cadenas hoteleras tienen una presencia cada vez más destacada, convirtiéndose en actores centrales del campo turístico. Según el estudio de Hernández Martín et al. (2018), el 52,9\% del total de plazas turísticas de la isla de Tenerife pertenecía en 2018 a una cadena hotelera (el 
4,6\% son internacionales, 33,4\% nacionales y 14,9\% locales). En cuarto lugar, más de la mitad de los establecimientos se construyeron entre los años sesenta y ochenta del siglo XX (gráfico 4). De forma que, en el mismo destino conviven establecimientos de distinta antigüedad, concentrándose los más antiguos con mayor probabilidad en ciertas zonas (Rodríguez-Rodríguez, 2017).

\section{Gráfico 4: Año de construcción de los establecimientos alojativos de Tenerife}

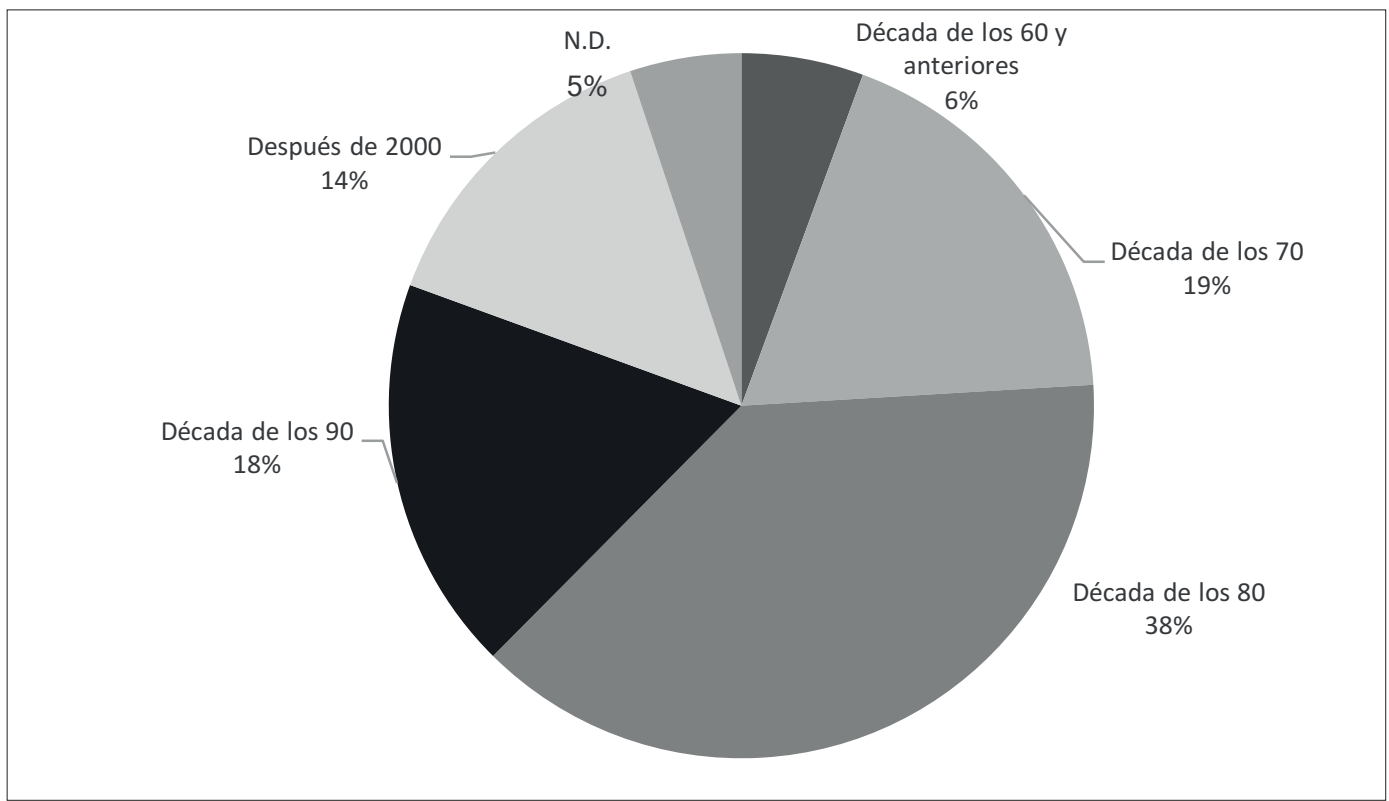

Fuente: Base de datos de REINVENTUR, 2017.

Así pues, el turismo en Tenerife no sólo se ha desarrollado y consolidado a lo largo de las últimas décadas, sino que lo ha venido haciendo en una dirección donde hoteles y cadenas hoteleras son cada vez más importantes, al tiempo que conviven con el subsector de establecimientos extrahoteleros; siendo los indicadores de rentabilidad claramente mejores en los primeros que en los segundos. Por otro lado, conviven en los destinos turísticos edificios modernos y antiguos, que afectan a la rentabilidad de éstos últimos.

\section{La complejidad normativa}

La importancia creciente de los hoteles y de las cadenas forma parte de un movimiento global al que las Islas Canarias no son ajenas (Alon et al., 2012; Rodríguez, 2002). Pero también tiene un contexto normativo, que refleja parcialmente el desequilibrio en la participación de los actores en la política turística canaria.

Dada la complejidad normativa desde los noventa hasta el presente, se subrayarán solo los procesos más relevantes. En primer lugar, la particular caracterización de los establecimientos extrahoteleros. Muchos de ellos no tienen un solo propietario, sino varios, a veces incluso uno por apartamento. Como plantean Simancas Cruz et al. (2017: 3-4): "las unidades alojativas extrahoteleras... se adquirieron antes de los años noventa, mediante el sistema de venta anticipada (sobre planos y con abono previo de parte del precio total), favorecido por la expectativa de autofinanciación y con el propósito de servir como fórmula de inversión de profesionales y fuente complementaria de ahorro local familiar... a diferencia del negocio hotelero, que requería la permanencia continuada en el mercado y la garantía de suficientes tasas de ocupación, los establecimientos de alojamiento extrahotelero presentaban menos costes, más diversidad de fórmulas de explotación y unos mayores márgenes de maniobrabilidad". 
Por ello se aprobó el denominado "principio de unidad de explotación" (Ley 7/1995, de 6 de abril, de Ordenación del Turismo de Canarias), fundamental para comprender el proceso posterior, mediante el cual muchos de estos establecimientos pudieron deslizarse hacia la residencialización o alquiler vacacional. Dicho principio determinó que la totalidad de los apartamentos debía comercializarse por una única empresa explotadora. Pero luego se flexibilizó -véase Disposición Transitoria Única de la Ley 5/1999, de 15 de marzo- (Simancas Cruz, 2016). Lo que favoreció la coexistencia en los complejos extahoteleros de planteamientos rentistas con los propiamente empresariales; y dando lugar a frecuentes situaciones conflictivas, por falta de acuerdos sobre mejoras, obras, precios o mecanismos de comercialización.

La explotación de la unidad alojativa disidente de la unidad de explotación turística se vió afectada por la Ley 4/2013 (4 de junio, de medidas de flexibilización y fomento del mercado del alquiler de viviendas, que ha modificado la Ley 29/1994 de 24 de noviembre, de Arrendamientos Urbanos, LAU en lo sucesivo); pues fue excluida de la posibilidad de arrendamiento privado de uso turístico. La unidad de explotación podía dividirse en subcomunidades de propietarios (tal como permite la ley 49/1960, de 21 de julio, sobre Propiedad Horizontal, y las modificaciones introducidas por la Ley 8/2013, de 26 de junio, de Rehabilitación, Regeneración y Renovación Urbana), con el consiguiente reparto de elementos comunes a una u otra; aplicar el régimen de propiedad horizontal en este caso genera distorsiones, al no estar previsto para estos supuestos, aunque es la institución más asimilable (Hermoso Mesa, 2014). Como se ha comentado, esta fragmentación de la propiedad hace que sus múltiples titulares no siempre coincidan en seguir la misma estrategia, sino que lleva en ocasiones al sumatorio de decisiones e intereses, a veces contrapuestas e individuales (Santana Turégano, 2005).

Ocurre en algunos de estos complejos con múltiples propietarios (con una o dos comunidades), que se va produciendo deterioro de los edificios, agravándose a medio plazo por la disminución de la rentabilidad y de la calidad de los servicios; ello provoca pérdida de competitividad y cuota de mercado, lo que suele contrarrestarse con la aplicación de una política agresiva de descuentos. Esto hace que se genere un círculo vicioso de precios ajustados y una modificación de los segmentos y patrones de demanda hacia menor calidad-diferenciación y precio. Si bien ello posibilita unos niveles mínimos de ocupación, no sucede lo mismo con los márgenes de beneficios; éstos siguen reduciéndose, repercutiendo sobre la disponibilidad de fondos económicos susceptibles de ser destinados a la modernización y mejora de las instalaciones y servicios turísticos. De ahí el descenso del valor de tales activos, decisivo para que determinados segmentos de población con un bajo poder adquisitivo puedan optar por la compra o alquiler de algunos de estos apartamentos. A partir de ahí se comprende la elevada rotación de la propiedad y el cambio de uso de los inmuebles turísticos a residenciales.

A esta particularidad de los complejos extrahoteleros canarios se une, en segundo lugar, la importancia de la "moratoria turística canaria" a partir de comienzos del siglo XXI, cuyo objetivo era limitar el crecimiento y cualificar y especializar la oferta, a través de la mejora de la modalidad y categoría del alojamiento turístico. Ello también ha favorecido indirectamente la conversión del apartamento turístico en alquiler vacacional, en cuanto ha priorizado la construcción de nueva oferta alojativa solo de hoteles de cuatro y cinco estrellas, en detrimento de la extrahotelera (Simancas Cruz, 2015). Se trata de una política pública de regulación del mercado a través del establecimiento de límites, ritmos y tipos de crecimiento de la oferta de alojamiento turístico, que, iniciada con la promulgación del Decreto 4/2001, por el que se acordó la formulación de las Directrices de Ordenación General y las del Turismo, continuó con la Ley 19/2003, por la que éstas se aprobaron, y se ha prolongado hasta la actualidad en virtud de las Leyes 6/2009, de 6 de mayo, de medidas urgentes en materia de ordenación territorial para la dinamización sectorial y la ordenación del turismo, 2/2013, de 29 de mayo, de renovación y modernización turística de Canarias, y 9/2015, de 27 de abril, que han modificado sustancialmente las pretensiones iniciales (García-Márquez, 2007; Rodríguez, 2014; Simancas Cruz, 2011; 2015; 2016; Simancas Cruz et al., 2017; Simancas Cruz et al., 2018; Villar-Rojas, 2016a; 2016b). Es importante subrayar que toda esta normativa que buscar favorecer la calidad en la oferta alojativa, no ha venido acompañada de mecanismos de mejora y modernización en el extrahotelero, pese a su importancia cuantitativa en el entramado productivo.

En tercer lugar, debe subrayarse que hay una situación paradójica en el caso del alquiler vacacional, ya desde su propia denominación. Lo habitual es que las viviendas tengan un carácter permanente, en detrimento del temporal, el que es inherente a los alojamientos turísticos (Bauzá Martorell, 2018). El artículo 42 b) de la Ley 7/1995 planteó que las unidades alojativas disidentes del principio de la unidad de la explotación sólo podrán ser alquilados o arrendados (contratos de temporada); por tanto, se permitía el uso residencial y, en ningún caso, el turístico. Ello se agravó con la LAU, que excluyó de su aplicación el arrendamiento privado de uso turístico. Esto se fundamentó en el hecho de que los 
arrendatarios son turistas (por tanto, consumidores), por lo que se trata de arrendamientos carentes de naturaleza civil.

El Decreto 113/2015, de 22 de mayo, por el que se aprueba el Reglamento de las viviendas vacacionales de la Comunidad Autónoma de Canarias, prohibió la explotación de las unidades alojativas fuera de la unidad de explotación bajo la fórmula del alquiler vacacional o similar. Este decreto constituye el preceptivo régimen específico y sectorial al que se alude en la LAU y que le permite hacerla operativa, pues, en el caso contrario los propietarios podían seguir utilizando la figura del arrendamiento por temporada. De esta manera, Canarias se ha equiparado a otras Comunidades Autónomas que han publicado regulaciones vinculadas a las tendencias de uso turístico de apartamentos y de viviendas residenciales, con el fin de evitar competencia desleal entre establecimientos turísticos autorizados y los particulares que destinan a hospedaje de turistas (Hermoso Mesa, 2014) y regular el arrendamiento vacacional como un tipo fuera del de temporada de la LAU. Asimismo, este Decreto excluye expresamente del ámbito de aplicación del Reglamento (artículo 3) a "las edificaciones ubicadas en suelos turísticos de las zonas turísticas o de las urbanizaciones turísticas, así como las viviendas ubicadas en urbanizaciones turísticas o en urbanizaciones mixtas residenciales turísticas"; en este sentido, se entiende como suelo turístico al definido como tal por cada uno de los Planes Territoriales de Ordenación Turística Insular. Lo anterior creó una situación (de incertidumbre) normativa en las Islas Canarias, recurrida por la Asociación Canaria del Alquiler Vacacional (ASCAV) y cuestionado por organismos como la Comisión Nacional de los Mercados y la Competencia, que en el Estudio sobre los nuevos modelos de prestación de servicios y la economía colaborativa (2015), instaba a promover la competencia; a lo que se suma la inexistencia de una normativa estatal básica. Así pues, la normativa ha favorecido la consolidación de los alojamientos hoteleros como símbolo de calidad, al tiempo que no ha creado fórmulas para la mejora del extrahotelero ni permitido la implementación del alquiler vacacional en muchas propiedades. Como veremos, ello no ha impedido su desarrollo.

\section{La irrupción del alquiler vacacional}

Las características del modelo turístico actual, donde conviven establecimientos hoteleros y extrahoteleros, sometidos a distintas tensiones y retos; favorecidos los hoteleros no solo por su mayor rentabilidad y recursos, sino también por una legislación que alienta su presencia, frente a complejos extahoteleros con rentabilidad inferior, menor atención normativa y tensiones internas. Estas características son el caldo de cultivo que va a condicionar el mapeado del alquiler vacacional en la isla.

Según el Observatorio de Turismo de Canarias en 2018 el número de plazas de alquiler vacacional era superior a 54.000 , lo que supondría el $26,5 \%$ del total de plazas alojativas en la isla de Tenerife (tabla I). Pues bien, teniendo en consideración el número de plazas y precios medios de vivienda vacacional y convencional por municipios, se observa que la oferta de vivienda vacacional está muy correlacionada con la existencia de plazas de oferta reglada $(r=0,9)$, y mucho menos con la tarifa media mensual por habitación (ADR) de apartamentos u hoteles (tabla II).

Dada la heterogeneidad urbana al interior de los municipios, también se ha realizado el análisis a nivel del microdestino, definido como espacios submunicipales homogéneos dentro del municipio turístico (Hernández-Martín et al., 2016; Rodríguez-Rodríguez, 2017), y tomando como fuente de datos Datahippo en noviembre 2019; aunque los casos son muy pocos (9), se observa que es más probable la presencia de plazas de alquiler vacacional cuanto mayor es el número de alojamientos turísticos convencionales $(r=0,71)$. Otras correlaciones son más moderadas: con las plazas ofertadas $(r=0,51)$, pernoctaciones $(\mathrm{r}=0,47)$, viajeros hospedados $(\mathrm{r}=0,46)$, y de signo negativo en el caso de la tarifa media mensual por habitación ADR $(r=-0,23)$, ingresos por habitación disponible RevPAR $(r=-0,26)$, y con la antigüedad de los establecimientos $(r=-0,26)$-tabla III-

Por tanto, la oferta se encuentra correlacionada con la infraestructura turística ya existente, porque los establecimientos alojativos convencionales son la base de la oferta de alquiler vacacional, tanto se haga el análisis a nivel municipal como de microdestino. De aquí se deriva que la normativa desarrollada por el Gobierno autonómico, orientada a restringir el alquiler vacacional en los municipios turísticos, ha sido poco efectiva; y que los espacios turísticos consolidados son un excelente caldo de cultivo para el alquiler vacacional, al contrario de lo que cabría esperar. En este sentido, la primera hipótesis (el alquiler vacacional aprovecha en Tenerife los espacios alojativos turísticos consolidados) se confirma. Y aunque en el caso de los microdestinos, el número de casos es pequeño, nos permite mantener la sospecha de que el alquiler vacacional está más vinculado a espacios de menor rentabilidad empresarial. Serían 
su caldo de cultivo. En este sentido, se interpretan estas correlaciones negativas con la antigüedad del establecimiento y su rentabilidad. Con lo que la segunda frase de la hipótesis sería considerada válida (especialmente los alojamientos extrahoteleros).

No obstante, indagando un poco más en los datos municipales (tabla I), se observa que la oferta de alquiler vacacional se está expandiendo por toda la isla, mientras que el turismo de hoteles y apartamentos está más concentrado en unos pocos municipios. Si se atiende a los municipios eminentemente turísticos (Puerto de la Cruz, Adeje, Arona) suman el 78,1\% de la oferta alojativa convencional; si le añade lo aportado por los municipios colindantes que han despegado turísticamente en las últimas décadas (Guía de Isora, Santiago del Teide, San Miguel de Abona y Granadilla de Abona), supone un $12 \%$ adicional. En total suman el $89,7 \%$ del total de plazas alojativas convencionales. Cuando se atiende a la geografía del alquiler vacacional, en los municipios turísticos se sitúa el 41,7\% de las plazas, añadiéndose un 19,3\% cuando se tienen en cuenta los municipios colindantes, llegando al 61\% del total de plazas de alquiler vacacional (Observatorio del Turismo de Canarias, datos 2018). La implicación es clara: el alquiler vacacional se expande más allá de los municipios propiamente turísticos (gráfico 5), colocando fuera de los mismos el 39\% de su oferta. Así, para este modelo de negocio la isla comienza a convertirse en el espacio turístico. Lo que conecta con las reivindicaciones de las asociaciones de representación del mismo, como veremos ahora.

Tabla III: Microdestinos según alojamientos, plazas de alquiler vacacional, plazas convencionales, pernoctaciones, viajeros, estancia media, ocupación, tarifas y antigüedad de los alojamientos convencionales

\begin{tabular}{|c|c|c|c|c|c|c|c|c|c|}
\hline & $\begin{array}{c}\text { Callao } \\
\text { Salvaje }\end{array}$ & $\begin{array}{c}\text { Playa } \\
\text { Paraíso }\end{array}$ & $\begin{array}{c}\text { Playa } \\
\text { de El } \\
\text { Duque }\end{array}$ & $\begin{array}{l}\text { Costa } \\
\text { Adeje }\end{array}$ & $\begin{array}{c}\text { Torviscas } \\
\text { - Fañabé } \\
\text { Alto }\end{array}$ & \begin{tabular}{|l} 
Las \\
Américas \\
- Adeje
\end{tabular} & \begin{tabular}{|l} 
Las \\
Américas \\
- Arona
\end{tabular} & $\begin{array}{c}\text { Los } \\
\text { Cristianos }\end{array}$ & $\begin{array}{c}\text { Costa } \\
\text { del } \\
\text { Silencio }\end{array}$ \\
\hline $\begin{array}{l}\text { Establecimientos } \\
\text { abiertos }\end{array}$ & 7 & 6 & 22 & 39 & 16 & 12 & 56 & 42 & 12 \\
\hline Plazas VV & 2.838 & 3.194 & 4.312 & 5.301 & 6.685 & 2.615 & 6.010 & 6901 & 4.078 \\
\hline $\begin{array}{l}\text { Plazas } \\
\text { ofertadas } \\
\text { convencionales }\end{array}$ & 2.219 & 4.642 & 11.999 & 18.690 & 4.835 & 5.371 & 27.699 & 11.754 & 2.339 \\
\hline Pernocataciones & 465.923 & 1.264 .517 & 3.440 .756 & 5.405 .379 & 1.221 .858 & 1.487 .280 & 7.418 .204 & 2.625 .714 & 538.452 \\
\hline $\begin{array}{l}\text { Viajeros } \\
\text { entrados }\end{array}$ & 64.987 & 196.613 & 472.137 & 693.848 & 159.718 & 201.969 & 938.950 & 356.413 & 72.719 \\
\hline Estancia media & 7,17 & 6,43 & 7,29 & 7,79 & 7,65 & 7,36 & 7,9 & 7,37 & 7,4 \\
\hline $\begin{array}{l}\text { Tasa de } \\
\text { ocupación por } \\
\text { plazas }\end{array}$ & 57,53 & 74,63 & 78,56 & 79,24 & 69,24 & 75,87 & 73,37 & 61,2 & 63,07 \\
\hline $\begin{array}{l}\text { Tarifa media } \\
\text { por habitación } \\
\text { mensual (ADR) }\end{array}$ & 66,32 & 112,15 & 146,65 & 90,13 & 59,46 & 95,36 & 84,67 & 86,48 & 68,1 \\
\hline $\begin{array}{l}\text { Ingresos por } \\
\text { habitación } \\
\text { disponible } \\
\text { (RevPAR) }\end{array}$ & 54,15 & 87,82 & 119,16 & 76,12 & 47,35 & 82,09 & 72,19 & 66,71 & 54,6 \\
\hline $\begin{array}{l}\text { Antigüedad } \\
\text { media respecto } \\
\text { a } 2019\end{array}$ & 35 & 22 & 20 & 26 & 28 & 45 & 32 & 32 & 35 \\
\hline
\end{tabular}

Fuente: DataHippo, elaboración propia (extraido en noviembre 2019) y base de datos de REINVENTUR. Todos los indicadores posteriores a las plazas ofertadas se refieren a los establecimientos convencionales. 
Gráfico 5: Distribución de plazas alojativas convencionales y de alquiler vacacional, según tipología de municipios, 2018

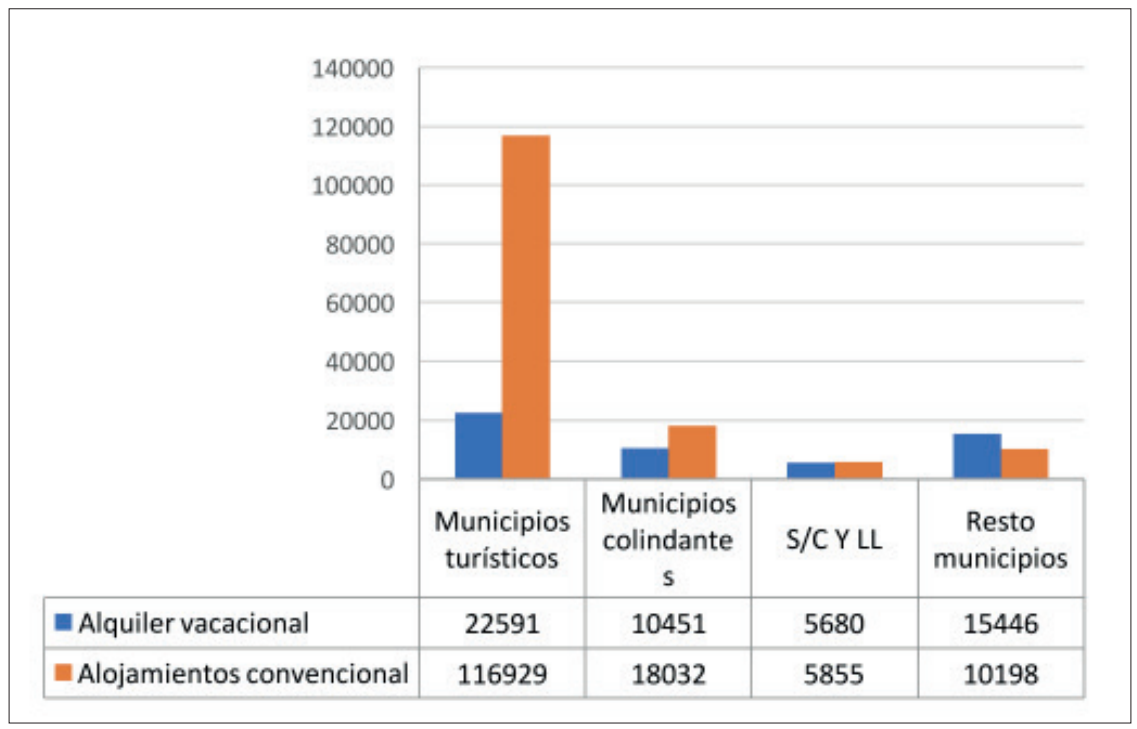

Fuente: Observatorio de Turismo de Canarias (se presentan separados los municipios de Santa Cruz de Tenerife y La Laguna por constituir el espacio metropolitano).

Antes de pasar al siguiente apartado, indicar que esta oferta de alquiler vacacional ya tiene una demanda. De la cual tenemos información gracias a los cambios metodológicos introducidos en 2018 en la Encuesta de Gasto Turístico del ISTAC. Para 2018, se observa que el 85,9\% de los turistas se alojaron en establecimientos convencionales (hoteleros, extrahoteleros, rurales, cruceros), el 5,9\% en una vivienda o habitación alquilada a un particular y el 8,1\% en una vivienda propia o a través de un intercambio gratuito. El peso relativo de la vivienda vacacional en 2018 indica que su presencia es todavía limitada, al menos en lo que respecta a la demanda. Así, en 2018 alrededor del 6\% de los turistas optó por el alquiler vacacional; por tanto, la llegada del alquiler vacacional tiene todavía poco impacto en el turismo convencional tinerfeño. Por lo que destaca un desfase entre oferta y demanda de alquiler vacacional, $26 \%$ de la oferta y $6 \%$ de la demanda. El perfil de personas hospedadas en este alojamiento difiere ligeramente del convencional, como se puede observar en la tabla IV. En general, estamos frente a un colectivo cuya característica central es su juventud, con una estancia superior y un nivel de repetición menor.

Tabla IV: Comparativa entre el comportamiento de turistas alojados en establecimientos convencionales y de alquiler vacacional, 2018

\begin{tabular}{|l|r|r|}
\hline & $\begin{array}{r}\text { Alojamientos } \\
\text { convencionales }\end{array}$ & 12,6 \\
\hline Motivos vacacionales & 62,2 & 5 \\
\hline Contratación de media pensión o todo incluido & 8,5 & 14,2 \\
\hline Estancia media & 66,6 & 11,8 \\
\hline Indice de repetición & 51,2 & 59,3 \\
\hline Porcentaje de jóvenes & 56,5 & 59,4 \\
\hline Ingresos anuales 50.000 euros o menos & 69 & 62 \\
\hline Estudios superiores & 64,3 & 79 \\
\hline Viajan en pareja y con hijos & & 71,8 \\
\hline
\end{tabular}

Fuente: Encuesta de Gasto Turístico, ISTAC. 


\section{La contienda discursiva en torno al alquiler vacacional}

Hasta la llegada del alquiler vacacional, el debate en torno al modelo turístico canario estaba organizado en torno a dos ejes discursivos: crecimiento versus moratoria en la construcción de nuevos alojamientos (y plazas alojativas), y calidad versus cantidad del modelo turístico; ambas cuestiones han funcionado como trasfondo en las iniciativas legislativas, y de la discusión política en torno a la importancia y peso del turismo en Canarias. La llegada del alquiler vacacional abre otro eje discursivo en torno al nivel de reglamentación apropiado para la actividad turística, lo que deriva en la definición de espacio turístico. De forma inevitable, este asunto afecta a los anteriores.

Del análisis de la prensa realizado, las instituciones y los políticos protagonizaban el 42\%, y la Asociación Canaria del alquiler vacacional (ASCAV) el 14\%. Las patronales siguen a continuación con el $11 \%$, el sector inmobiliario con el 5,6\%, completando el escenario la Asociación de Municipios Turísticos, la Plataforma de afectados por la ley turística, la Plataforma Canaria por un desarrollo sostenible, y colectivos vecinales, con una noticia cada uno. Este protagonismo de instituciones y organizaciones políticas se debe al contexto: a comienzos de 2019 el Parlamento de Canarias trató de aprobar una nueva regulación del Alquiler Vacacional a través de una modificación de la Ley de Renovación y Modernización Turística. Este proceso quedó paralizado tras un informe negativo del Consejo Consultivo de Canarias. De ahí, esta centralidad política. También el que varios municipios importantes (desde el punto de vista demográfico o turístico) hubiesen puesto en marcha la negociación y aprobación de ordenanzas sobre alquiler vacacional.

En la mayoría de estas noticias, la atención se centra en los principios y criterios de la regulación del alquiler vacacional. En el discurso de representantes políticos y de ASHOTEL es unánime la necesidad de la regulación, aunque sin especificar, al igual que sucede respecto a los impactos. Como cabría esperar, la Asociación Canaria del alquiler vacacional (ASCAV) defiende argumentos menos reguladores que los anteriores actores. Los argumentos a favor de la regulación apelan al interés general y de las condiciones de vida de los canarios, al impacto sobre el incremento de los precios de la vivienda para los residentes, y para evitar la irregularidad y la turismofobia; incluso un alcalde de un importante municipio turístico valora como positivo el que pueda favorecer mediante la regulación la explotación de apartamentos turísticos obsoletos. Solo de forma minoritaria se apela a un alto nivel de regulación por la degradación y pérdida de identidad de los barrios (Ecologistas en Acción, Plataforma Canaria para un Territorio Sostenible, Barrio para vivir). La patronal demanda igualdad de condiciones para todas las empresas turísticas, en cuanto a exigencias normativas y tributarias, a favor de la "competencia sana".

Para la ASCAV, el problema es el exceso de regulación, presentándose como defensora de los pequeños propietarios, del crecimiento económico derivado del alquiler vacacional, de los emprendedores sometidos a la inseguridad jurídica, y de la regeneración urbanística y turística. La prohibición del alquiler vacacional supone un rechazo del crecimiento turístico. Por ello, en algunas declaraciones se presentan como portavoces de miles de familias canarias perjudicadas por la normativa restrictiva de Gobierno y ayuntamientos. Para toda esta narrativa se apoyan en las sucesivas resoluciones del Tribunal Supremo anulando preceptos sobre alquiler vacacional y en los retrasos en los procesos de inscripción de viviendas vacacionales. Apoyan algunos de estos planteamientos la Federación de Desarrollo Empresarial de Canarias y la Asociación de inmobiliarias del sur de Tenerife.

Así, habría un continuum con respecto a la regulación, desde colectivos que se inclinan por la prohibición, hasta aquellos que defienden la desregulación. Lo que para algunos es regulación, para otros es prohibición encubierta. Para algunos es alegal, lo que para otros es retraso en la inscripción de viviendas. Pero, además, hay que subrayar una idea fundamental: la defensa de la isla completa como turística, alterando el supuesto con el que la normativa y los actores (empresariales y públicos) han venido trabajando en las últimas décadas, la delimitación del espacio insular entre turístico y residencial. Una actuación reciente e ilustrativa es la presentación pública el 4 de febrero de 2020 del Círculo Turístico de Canarias, lobby en el que se incluye ASCAV, pero también la Federación Provincial de Entidades de la Construcción de Santa Cruz de Tenerife (FEPECO), la Asociación Profesional de Expertos Inmobiliarios (APEI), Tenerife South Real Estate Association, la Asociación de Empresarios de Arona (Tenerife), la Federación de Autónomos de Canarias y la empresa Autobuses Mesa (La Gomera). Entre otras propuestas realizan las siguientes: supresión de "limitaciones injustificadas" al alquiler vacacional y declaración por parte del Gobierno autonómico de todos los municipios canarios como turísticos (cuando en la actualidad se consideran como tales Puerto de la Cruz, Adeje, Arona y Guñia de Isora). 
El alquiler vacacional es defendido por ASCAV, mientras que la patronal hotelera y extrahotelera y la mayoría de los representantes políticos se inclinan por algún tipo de regulación. En esta batalla discursiva es central la dimensión regulatoria. Además, interesa subrayar que la defensa del alquiler vacacional ha encontrado en ASCAV un actor puesto a la defensa de su expansión, acompañado de algunas asociaciones empresariales y agencias inmobiliarias. Y que en sus planteamientos se quiebra el supuesto de que el turismo y los residentes se ubican en distintos espacios insulares. Ahora cualquier lugar de la isla puede ser considerado turístico. Por parte del empresariado hotelero, hay en sus discursos una apuesta por la renovación edificatoria y por la innovación, y también una demanda de reglamentación del nuevo modelo de negocio del alquiler vacacional. Las referencias a la innovación señalan a los edificios (deben renovarse periódicamente), al modelo de negocio (digitalización, estructuras horizontales y colaborativas) y a la relación con el entorno (sostenibilidad y de colaboración activa con los actores del destino, coopetición). Por tanto, no solo la oferta de alquiler vacacional se expande más allá de los municipios turísticos, apoyada en los mecanismos de comercialización de las plataformas digitales de alojamientos, sino que a nivel local surgen actores nuevos (ASCAV), que se asocian a otros ya existentes, como empresas inmobiliarias, y que desarrollan un discurso donde la idea de la isla como espacio turístico es central.

\section{Reflexiones finales}

Como ocurre en otros muchos lugares, el alquiler vacacional se está expandiendo en la isla de Tenerife. Pasa a hacerlo en un contexto específico marcado por varias dimensiones: la estructura alojativa turística convencional, los cambios normativos y el equilibrio de fuerzas entre actores, que favorece a hoteleros frente extrahoteleros. Éstos, con una menor rentabilidad que hoteles y cadenas, encuentran en el alquiler vacacional una oportunidad que las legislaciones anteriores y sus propios 'vicios' históricos no les habían proporcionado. La legislación en las últimas décadas ha favorecido a la oferta hotelera, nunca ha apostado por el extrahotelero, siguiendo una filosofía marcada por la equiparación de calidad con más estrellas hoteleras. Esta legislación tampoco ha podido resolver los problemas derivados de la multiplicidad de propietarios en muchos complejos de apartamentos y de la construcción de edificios en cuya orientación siempre han estado mezcladas múltiples motivaciones (uso propio, uso turístico, alquiler temporal...). La unidad de explotación no ha funcionado en muchos establecimientos, lo que se añade a la perspectiva rentista de los propietarios. Además, el ahorro de muchas familias se orienta principalmente a la adquisición de inmuebles, lo que viene a ser una constante institucional-cultural española al considerar la inversión en inmuebles como la mejor de las opciones (Bernardos Domínguez, 2009; Lois González et al., 2016; Serrano Martínez, 2003).

El campo económico turístico tinerfeño es complejo y las fuerzas que dinamizan la renovación edificatoria están desigualmente repartidas. A lo largo de los últimos cuarenta años, la renovación ha tenido lugar primero a través de la edificación de nuevos hoteles de nivel medio-alto, por la sustitución de extrahotelero por hotelero, y más recientemente por la renovación edificatoria. Esta última acción está impulsada por empresarios y directivos más profesionalizados, de segunda o terceras generaciones que conviven en un entorno empresarial diverso. Los recursos y capacidades están desigualmente repartidos, pues son las cadenas hoteleras y hoteles en general los que cuentan con más recursos para hacer frente a la renovación; al tiempo que la administración favorece a estos grupos. Sin embargo, en este entramado productivo persisten complejos alojativos con bajas rentabilidades, que han encontrado en el alquiler vacacional una nueva oportunidad para mantenerse en el mercado turístico.

Favoreciendo que haya una correlación entre espacios turísticos y oferta de plazas de alquiler vacacional. Ello no obsta para que dicha oferta se expanda a todo el territorio insular.

Y además, esta dinámica no ocurre en el vacío. Surgen asociaciones como ASCAV con mucha presencia mediática y apoyo de otras organizaciones (inmobiliarias, sobre todo), que defienden el alquiler vacacional y promueven la idea de la isla como espacio turístico. El mercado turístico está polarizado, pues el crecimiento de las cadenas hoteleras y de los hoteles individuales orienta hacia la calidad del alojamiento, con una cadencia y recursos a los que parte del sector extrahotelero no puede aspirar. El alquiler vacacional les ha dado una nueva oportunidad.

$\mathrm{Al}$ menos de momento, y de forma derivada, la irrupción del alquiler vacacional parece ampliar los límites de los espacios turísticos. Hasta ahora, la mayor parte de la oferta ha estado concentrada en unos pocos municipios, mientras que se detecta una progresiva extensión más allá de dicho espacio. Quedan para análisis posteriores, una primera cuestión que solo el tiempo determinará, y es si se consolida 
esta nueva oferta o si, por el contrario, la baja tasa de ocupación que podría preverse del desfase entre oferta y demanda reconduce los espacios turísticos a fronteras semejantes a las actuales. Una segunda pregunta a responder tendrá que ver con las relaciones entre los actores implicados en este campo. La preponderancia del subsector hotelero ha dificultado que otros ámbitos alojativos pueden beneficiarse mediante normas u otro tipo de recursos; con el alquiler vacacional, nuevos actores irrumpen en el campo turístico. Parece confirmarse que la estructura y características del actual modelo turístico afecta a la geografía de la oferta del alquiler vacacional. En definitiva, próximas investigaciones habrán de abordar qué definición de espacio turístico y mecanismos de regulación del mismo prevalecerán, y a qué actores beneficiará; y si la opción por el alquiler vacacional proporciona una nueva herramienta de posicionamiento en el mercado a los establecimientos extrahoteleros que han apostado por el mismo.

\section{Bibliografía}

Agarwal, Sheela (1997). "The resort cycle and seaside tourism: an assessment of its applicability and validity". Tourism Management, 18(2): 65-73. https://doi.org/10.1016/S0261-5177(96)00102-1

Agarwal, Sheela (2002). "Restructuring seaside tourism: The Resort Lifecyle". Annals of Tourism Research, 29(1): 25-55. https://doi.org/10.1016/S0160-7383(01)00002-0

Agarwal, Sheela (2006). "Coastal Ressort Restructuring and the TALC". En: R.W. Butler, The Tourism Area Life Cycle. Vol.1 Applications and Modifications. Channel View Publications. https://doi. org/10.21832/9781845410308-022

Agarwal, Sheela y Shaw, Gareth (2007). Managing coastal tourism resorts: a global perspective. Clevedon (UK), Buffalo (NY): Channel View Publications. https://doi.org/10.21832/9781845410742

Alon, I., Ni, L., \& Wang, Y. (2012). Examining the determinants of hotel chain expansion through international franchising. International Journal of Hospitality Management, 31(2), 379-386. https:// doi.org/10.1016/j.ijhm.2011.06.009

Bashir, M., \& Verma, R. (2016). Airbnb disruptive business model innovation: Assessing the impact on hotel industry. International Journal of Applied Business and Economic Research, 14(4), 2595-2604.

Bauzá Martorell, Felio José (2018). "Intervención administrativa en la vivienda turística vacacional". Revista española de derecho administrativo, 189: 313-346.

Bericat, Eduardo (1998). La integración de los métodos cuantitativo y cualitativo en la investigación social. Barcelona: Ariel.

Bernardos Domínguez, Gonzalo (2009). "Creación y destrucción de la burbuja inmobiliaria en España”. Información Comercial Española. Revista de Economía ICE, 850: 23-40.

Butler, Richard (1980). "The concept of a tourist area cycle of evolution. Implications for management of resources". Canadian Geographer, 24(1): 5-12. https://doi.org/10.1111/j.1541-0064.1980.tb00970.x

Butler, Richard (2006). The Tourism Area Life Cycle. Vol.1 Applications and Modifications. Channel View Publications. https://doi.org/10.21832/9781845410278

Butler, Richard (2012): “Mature Tourist Destinations: can we recapture and retain the magic?". En: F. Vera Rebollo e I. Rodríguez Sánchez (eds.): Renovación y restructuración de destinos en áreas costeras. Marco de análisis, procesos, instrumentos y realidades. Colección Desarrollo Territorial, 11. Universitat de València, pp. 19-36.

Butler, Richard y Russell, Roslyn (eds.) (2010). Giants of tourism. Wallingford: CABI. https://doi. org/10.1079/9781845936525.0000

Cooper, Chris y Jackson, Stephen (1989). "Destination life cycle: the isle of Man case study". Annals of Tourism Research, 16(3): 377-398. https://doi.org/10.1016/0160-7383(89)90051-0

Fligstein, Neil (2013). "Understanding stability and change in fields". Research in Organizational Behavior, 33: 39-51. https://doi.org/10.1016/j.riob.2013.10.005

Fligstein, Neil y McAdam, Doug (2011). “Toward a general theory of strategic action fields". Sociological Theory, 29(1): 1-26. https://doi.org/10.1111/j.1467-9558.2010.01385.x

Fligstein, Neil y McAdam, Doug (2012). A theory of fields. New York: Oxford University Press. https://doi.org/10.1093/acprof:oso/9780199859948.001.0001

Garau-Vadell, Joan B., Gutiérrez-Taño, Desiderio y Díaz-Armas, Ricardo (2019). "Residents' support for P2P accommodation in mass tourism destinations". Journal of Travel Research, 58(4): 549-565. https://doi.org/10.1177/0047287518767067

Garay, Lluís y Cànoves, Gemma (2011). "Life cycles, stages and tourism history. The Catalonia (Spain) experience". Annals of Tourism Research, 38(2): 651-671. https://doi.org/10.1016/j.annals.2010.12.006 
García-Márquez, Faustino (2007). "La nueva generación de Directrices Territoriales/Turísticas y la Sostenibilidad: la experiencia canaria". Estudios Turísticos, 172-173: 89-96.

Goldstone, Jack A. y Useem, Bert (2012). "Putting values and institutions back into the theory of strategic action fields". Sociological Theory, 30(1): 37-47. https://doi.org/10.1177/0735275112437161

Groizard, José Luis y Nilsson, William (2017). Mito y realidad del alquiler vacacional en las Islas Baleares. Análisis y recomendaciones de política turística. Universitat de les Illes Balears, Departament d'Economía Aplicada, número 84.

Guttentag, D. (2015). Airbnb: disruptive innovation and the rise of an informal tourism accommodation sector. Current issues in Tourism, 18(12), 1192-1217.https://doi.org/10.1080/13683500.2013.827159

Hall, Michael y Müller, Dieter (eds.) (2018). The Routledge handbook of second home tourism and mobilities. Routledge. https://doi.org/10.4324/9781315559056

Hermoso Mesa, Diego (2014): "La calificación turística de la parcela y del inmueble. Constancia en el registro de la propiedad. Ley 2/2013, de Renovación y Modernización Turística de Canarias". Boletín del Colegio de Registradores de España, XLIX (11): 2108-2120.

Hernández Martín, Raúl et al. (2018). Importancia de las cadenas hoteleras en Tenerife. Cátedra de Turismo de la Universidad de La Laguna.

Hernández-Martín, Raúl (2016). "Impactos económicos del turismo”. En: M. Simancas Cruz y E. Parra (coords.), ¿Existe un modelo turístico canario? Santa Cruz de Tenerife: Promotur Turismo de Canarias, colección Horizonte del Turismo en Canarias/ULL Opina.

Hernández-Martín, Raúl et al. (2016). "Identifying micro-destinations and providing statistical information: a pilot study in the Canary Islands". Current Issues in Tourism, 19(8): 771-790. https://doi. org/10.1080/13683500.2014.916657

Lois González, Rubén C., Piñeira Mantiñan, María José y Vives Miró, Sònia (2016). "El proceso urbanizador en España (1990-2014): una interpretación desde la geografía y la teoría de los circuitos de capital". Scripta Nova. Revista electrónica de geografía y ciencias sociales, 20 (539). https://doi. org/10.1344/sn2016.20.16793

Ma, Mulan y Hassink, Robert (2013). "An evolutionary perspective on tourism area development". Annals of Tourism Research, 41: 89-109. https://doi.org/10.1016/j.annals.2012.12.004

Marrero-Rodríguez, J.R. (2019). "Los campos de acción estratégica como instrumento analítico de los destinos turísticos maduros: Tenerife (Islas Canarias)". En A. Alvarez Sousa, A. Mantecón, I. Puertas-Cañaveral (eds.), Sociología del turismo. Madrid, Centro de Investigaciones Sociológicas, 2019.

Mikhalkina, T., \& Cabantous, L. (2015). Business model innovation: How iconic business models emerge. Advances in Strategic Management, 33(1), 59-95. https://doi.org/10.1108/S0742-332220150000033024

Onete, Cristian Bogdan, Pleşea, Doru., and Budz, Sonia (2018). Sharing Economy: Challenges and Opportunities in Tourism. Amfiteatru Economic, 20(12), 998-1015. https://doi.org/10.24818/EA/2018/ S12/998

Oppermann, Martin (1998). "What is new with the resort cycle? Comment". Tourism Management, 19(2): 179-180. https://doi.org/10.1016/S0261-5177(97)00110-6

Oreja, Juan Ramón; Parra, Eduardo y Yanes, Vanessa (2008). "The sustainability of island destinations: Tourism area life cycle and teleological perspectives. The case of Tenerife" Tourism Management, 29(1): 53-65. https://doi.org/10.1016/j.tourman.2007.04.007

Padrón-Marrero, David y Rodríguez-Martín, José Ángel (2015). Economía de Canarias: dinámica, estructura y retos. Valencia: Tirant lo Blanch.

Perles Ribes, José Francisco et al. (2018). "The rental prices of the apartments under the new tourist environment: A hedonic price model applied to the Spanish sun-and-beach destinations". Economies, 6(2): 23. https://doi.org/10.3390/economies6020023

Ravenscroft, Neil y Hadjihambi, Ion (2006). "The implications of Lamarckian theory for the TALC model". En: R.W. Butler (ed.), The Tourism Area Life Cycle. Vol.2. Conceptual and Theoretical Issues. Clevedon: Channel View Publications. https://doi.org/10.21832/9781845410308-018

Rodríguez, A. R. (2002). Determining factors in entry choice for international expansion. The case of the Spanish hotel industry. Tourism Management, 23(6), 597-607. https://doi.org/10.1016/S0261-5177(02)00024-9

Rodríguez, Miguel Ángel (2014). "La moratoria turística en las Islas Canarias: asignaturas pendientes". ACE: Architecture, City and Environment = Arquitectura, Ciudad y Entorno, 9 (25): 467-492. https:// doi.org/10.5821/ace.9.25.3636

Rodríguez-Rodríguez, Yurena (2017). Delimitación de destinos turísticos locales con fines estadísticos a partir de criterios de oferta. Tesis doctoral. Universidad de La Laguna. 
Santana Turégano, Manuel Ángel (2005). “Turismo, empleo y desarrollo”. Papers: Revista de Sociología, 77: 0079-104. https://doi.org/10.5565/rev/papers/v77n0.932

Serrano Martínez, José María (2003). "Las viviendas de segunda residencia en la sociedad de "bienestar". El caso de un país turístico: España". Cuadernos de Turismo, 12: 53-75.

Simancas Cruz, Moisés (2011). "El fracaso de la renovación de áreas turísticas consolidadas de litoral a través de la sustitución de la oferta de alojamiento obsoleta: la experiencia de las Islas Canarias". Cuadernos de Turismo, 27: 875-906.

Simancas Cruz, Moisés (2015). La moratoria turística de Canarias. La reconversión de un destino turístico maduro desde la Ordenación del Territorio. La Laguna: Servicio de Publicaciones de la Universidad de La Laguna.

Simancas Cruz, Moisés (2016). "La política canaria de renovación de las áreas turísticas del litoral". En: M. Simancas Cruz y E. Parra (coords.), ¿Existe un modelo turístico canario?. Santa Cruz de Tenerife: Promotur Turismo de Canarias, colección Horizonte del Turismo en Canarias/ULL Opina.

Simancas Cruz, Moisés (2016): "Efectos y defectos del uso residencial y el alquiler vacacional de los establecimientos de alojamiento extrahoteleros". En: M. Simancas Cruz y J. Mañoso Valderrama (coords.), La residencialización de las áreas turísticas de Canarias. Santa Cruz de Tenerife: Promotur Turismo Canarias / UIMP.

Simancas Cruz, Moisés; Peñarrubia Zaragoza, María Pilar y Temes Cordovez, Rafael (2018). "La calificación urbanística del suelo: cuestión clave en la prohibición del alquiler vacacional en las áreas turísticas de litoral de Canarias". Cuadernos Geográficos 57(1): 177-196. https://doi.org/10.30827/ cuadgeo.v57i1.5666

Simancas Cruz, Moisés; Temes Cordovez, Rafael y Peñarrubia Zaragoza, María Pilar (2017). "El alquiler vacacional en las áreas turísticas de litoral de Canarias". Papers de Turisme, 60: 1-24.

Villar-Rojas, Francisco José (2016a). "El marco normativo de definición del modelo turístico canario". En: M. Simancas Cruz y E. Parra (coords.), ¿Existe un modelo turístico canario? Santa Cruz de Tenerife: Promotur Turismo de Canarias, colección Horizonte del Turismo en Canarias/ULL Opina.

Villar-Rojas, Francisco José (2016b). "Crónica sobre la construcción de un marco legal para la renovación del espacio turístico en Canarias”. El Consultor Urbanístico, 138.

Wegmann, J., \& Jiao, J. (2017). Taming Airbnb: Toward guiding principles for local regulation of urban vacation rentals based on empirical results from five US cities. Land Use Policy, 69, 494-501. https:// doi.org/10.1016/j.landusepol.2017.09.025

Yrigoy, Ismael (2017). "Airbnb en Menorca: ¿Una nueva forma de gentrificación turística? Localización de la vivienda turística, agentes e impactos sobre el alquiler residencial”. Scripta Nova. Revista Electrónica de Geografía y Ciencias Sociales, 21.https://doi.org/10.1344/sn2017.21.18573

\section{Notas}

Esta publicación nace del proyecto "Tecnología de Información Geográfica aplicadas al análisis y diseño de propuestas innovadoras para la renovación de los alojamientos turísticos”, dirigido por Moisés Simancas Cruz. 